\title{
Association of placental chorangiosis with pregnancy complication and prenatal outcome: a case-control study
}

Homeira Vafaei ${ }^{1,2}$, Zinat Karimi ${ }^{1,2}$, Mojgan Akbarzadeh-Jahromi ${ }^{1,3^{*}}$ and Fatemeh Asadian ${ }^{4}$

\begin{abstract}
Background: Chorangiosis is a vascular change involving the terminal chorionic villi in the placenta. It results from longstanding, low-grade hypoxia in the placental tissue, and is associated with such conditions as intrauterine growth restriction (IUGR), diabetes, and gestational hypertension in pregnancy. Chorangiosis rarely occurs in normal pregnancies. However, its prevalence is $5-7 \%$ of all placentas from infants admitted to newborn intensive care units. The present study was aimed at determining the association of chorangiosis with pregnancy complications and perinatal outcomes.

Methods: In this case-control study, 308 chorangiosis cases were compared with 308 controls (with other diagnoses in pathology) in terms of maternal, placental, prenatal, and neonatal characteristics derived from the medical records of participants retrospectively. $R$ and SPSS version 22 software tools were used, and the statistical significance level was considered 0.05 for all the tests.

Results: Preeclampsia, diabetes mellitus, maternal hemoglobin, maternal hematocrit, C/S, oligohydramnios, fetal anomaly, dead neonates, NICU admissions were significantly higher in the chorangiosis group $\mathrm{OR}=1.6,3.98,1.68$, $1.92,2.1,4.47,4.22,2.9,2.46$, respectively ( $p$-value $<0.05$ for all). Amniotic fluid index, birth weight, cord PH amount, 1st, and 5th Apgar score was lower in the chorangiosis group $\mathrm{OR}=0.31,1,0.097,0.83,0.85$, respectively ( $p$-value $<$ 0.05 for all). Moreover, fundal placenta, retro placental hemorrhage, perivillous fibrin deposition, calcification, and acute chorioamnionitis were higher in the chorangiosis group $\mathrm{OR}=2.1,11.8,19.96,4.05$, and 6.38 respectively, $(p$ value $<0.05)$. There was a high agreement between the two pathologists, and the power of the study was estimated at $99 \%$.
\end{abstract}

Conclusion: Although chorangiosis is an uncommon condition, it is associated with a higher incidence of perinatal and neonatal morbidity and mortality. Therefore, it should be considered an important clinical sign of adverse pregnancy outcomes and should be reported in the pathology evaluation.

Keywords: Chorangiosis, Perinatal outcomes, Neonatal outcomes

\footnotetext{
* Correspondence: akbarzadeh@sums.ac.ir; mojganakbarzadeh@yahoo.com

${ }^{1}$ Maternal-Fetal Medicine Research Center, Shiraz University of Medical

Sciences, Shiraz, Iran

${ }^{3}$ Pathology Department, School of Medicine, Shiraz University of Medical

Sciences, Postal code/ P.O. Box: 34786-71946, Shiraz, Iran

Full list of author information is available at the end of the article
}

(c) The Author(s). 2021 Open Access This article is licensed under a Creative Commons Attribution 4.0 International License, which permits use, sharing, adaptation, distribution and reproduction in any medium or format, as long as you give appropriate credit to the original author(s) and the source, provide a link to the Creative Commons licence, and indicate if changes were made. The images or other third party material in this article are included in the article's Creative Commons licence, unless indicated otherwise in a credit line to the material. If material is not included in the article's Creative Commons licence and your intended use is not permitted by statutory regulation or exceeds the permitted use, you will need to obtain permission directly from the copyright holder. To view a copy of this licence, visit http://creativecommons.org/licenses/by/4.0/. The Creative Commons Public Domain Dedication waiver (http://creativecommons.org/publicdomain/zero/1.0/) applies to the data made available in this article, unless otherwise stated in a credit line to the data. 


\section{Background}

The placenta, as the largest organ of fetal origin, plays an essential role in fetal development and function from implantation to delivery and affects maternal function during pregnancy. In this regard, histological examination of the placenta provides a wide range of specific information about the nature, cause, and timing of injuries to both the fetus and mother in cases with poor obstetrical outcomes [1-3].

Since oxygen transfer between the mother and fetus is just made through the placenta, its pathological feature indicates current and/or previous chronic fetal hypoxia $[2,4,5]$. Sometimes, villous capillary lesions of the placenta develop as compensatory attempts to improve chronic fetal hypoxia. These lesions form a heterogeneous group of possibly interrelated alterations $[6,7]$.

Normal chorionic villi should contain fewer than five capillaries in ten high-power microscopic fields, and larger numbers defined as hypervascularity. Extreme villous hypervascularity is known as chorangiosis, characterized by more than 10 capillaries in more than 10 terminal chorionic villi in several areas of the placenta [8].

Chorangiosis is found in 5-7\% of all placentas from infants admitted to newborn intensive care units (NICU) [9], and it has to do with increased neonatal morbidity as well as mortality. Furthermore, the prevalence of chorangiosis is higher when some other acute or chronic hypoxia-related placental lesions are present [10-14].

There are limited studies on chorangiosis; its pathogenesis is not precisely exact but believed to result from chronic hypoperfusion and hypoxia [9]. Various maternal, fetal, and placental conditions have been suggested as playing a role in the development of chorangiosis [15], which can be classified into three major categories, namely preuterine, uterine and postuterine patterns of chronic hypoxic placental injury $[5,16]$. Preuterine conditions include maternal hypoxemia resulting from low oxygen pressure in the ambient environment (e.g., pregnancies at high altitudes), maternal anemia, high levels of air pollution, maternal smoking, multiple pregnancies, and maternal diabetes mellitus. The uterine pattern of chronic hypoxic placental injury also intervenes in the development of choragiosis. By uterine here, we mean the maternal portions of the uteroplacental circulation, mainly its myometrial and decidual segments, which are the contributing factors to pathologic placental changes. Preeclampsia and late-onset fetal growth retardation are typical examples of the uterine pattern of hypoxia. Finally, primary villous changes, typically referred to as the postuterine (postplacental) pattern of chronic hypoxic placental injury, result in reduced intake of oxygen from the intervillous space. This is often seen in subsets of fetal growth retardation and preeclampsia, and fetal thrombotic vasculopathy (only focally) $[5,16]$.
Given that chorangiosis is associated with fetal, maternal, and placental disorders, this study is designed to review 308 cases with chorangiosis over a 4-year period to determine the association of chorangiosis with perinatal outcomes.

\section{Material \& Methods \\ Study design}

This is a case-control study conducted through April 2014 to March 2018. The cases and controls were chosen from the placentas sent to the Pathology Department of Hazrat Zeinab Hospital, affiliated to Shiraz University of Medical Sciences. All placentas had been sent for histopathological examination at the discretion of obstetricians due to high-risk pregnancy, operative delivery, poor condition of the neonate, or gross placental abnormalities.

Selection criteria: Inclusion criteria included the placentas of women aged 15-50 years with gestational age $\geq$ 20 weeks and singleton pregnancy. Exclusion criteria included incomplete medical records.

\section{Placenta tissue sampling}

All the placentas which arrived at the pathology department were weighted after removal of the cords and membranes. Multiple sections were taken from each placenta, including two sections from the umbilical cord and one from the membrane roll, at least three sections from placental parenchyma (one near umbilical cord insertion and two from central two-third). The tissue was fixed in $10 \%$ buffered formalin, embedded in paraffin, and stained with hematoxylin-eosin. Chorangiosis was diagnosed based on the criteria established by Altshuler in 1984 [8], defined as the presence of a minimum of ten villi, each with ten or more vascular channels present in ten or more non-infarcted areas of at least three different placental regions when using an $\times 10$ ocular Lenz. Additionally, other placental pathologies such as calcification, fibrin deposition, retroplacental hemorrhage, and membrane and cord pathology were checked [17]. Reporting of the chorangiosis was done by two pathologists who read the pathology slides and decided on the presence or absence of chorangiosis in the placentas.

\section{Data collection}

Maternal, placental, prenatal, and neonatal features of the participants were retrospectively derived from the medical records of mothers whose placentas had been sent to the pathology department. The variables were maternal features, including maternal age, gestational age, gravid, parity, history of background diseases, hematological indices, and rout of delivery. Fetal features included fetal growth (IUGR, is an estimated fetal weight below the 10th percentile for gestational age) [18], 
amniotic fluid index, fetal anomaly, and abnormal fetal karyotype. Neonatal features included gender, survival, birth weight $(\mathrm{kg})$, cord $\mathrm{PH}$, first and fifth Apgar scores, and NICU admission. Pathology features included placenta pathology, decidua pathology, membrane pathology, and cord pathology. To control the potential confounding effects of maternal age, gestational age, gravid, and parity, adjusted regression tests were applied in the analysis phase.

\section{Statistical analyses}

Regarding type I error and type II error equal to 0.05 and 0.20 , respectively, and based on IUGR association with chorangiosis and the case-control ratio of one, the minimum number of sample size in each group was determined using Cochran's Sample Size formula. To control the exogenous variables not recorded for the patients as well as the latent confounders, and enhance the power of the study and validity of the results, an attempt was made to take all the cases occurring during April 2014 and March 2018 from a total of 2500 placentas. Finally, at the end of the analysis, a power analysis was performed to estimate the power of the study based on the study results using the followings:

$$
\text { power }=\phi\left\{\frac{\Delta}{\sqrt{p_{1} q_{1} / n_{1}+p_{2} q_{2} / n_{2}}}-z_{1-\alpha / 2} \frac{\sqrt{\overline{p q}\left(1 / n_{1}+1 / n_{2}\right)}}{p_{1} q_{1} / n_{1}+p_{2} q_{2} / n_{2}}\right\}, q=1-p, \bar{p}=\frac{p_{1}+k p_{2}}{1+k}
$$

$\mathrm{p} 1, \mathrm{p} 2=$ proportion of groups 1 and 2 , respectively. $\Delta=|\mathrm{p} 2-\mathrm{p} 1|=$ absolute difference between two proportions.

$\mathrm{n} 1=$ sample size for group 1.

$\mathrm{n} 2$ = sample size for group 2.

$\alpha=$ probability of type I error.

$\mathrm{Z}=$ critical $\mathrm{Z}$ value for a given $\alpha$.

$\mathrm{K}=$ ratio of sample size for group 2 to group 1 .

$\Phi()$ = function converting a critical $\mathrm{Z}$ value to power.

Continuous and discreet variables were described by mean \pm standard deviation and frequency and relative frequency, respectively. Kolmogorov-Smirnov test of normality, t-test, $\mathrm{X}^{2}$ test, and logistic regression adjusted on maternal age, gestational age, gravid, and parity were used. Kappa statistics were calculated to evaluate how accurate and valid the reporting of the choriongiosis was. Moreover, a power analysis was applied to estimate the power of the study. R and SPSS version 22 software tools were used, and the statistical significance level was considered 0.05 for all the tests.

\section{Results}

There were 308 cases diagnosed with chorangiosis, and 308 other placenta complications controls in the study. The maternal age and gestational age were lower in the chorangiosis group $(29.4 \pm 5.8,30.3 \pm 5.48$ and $35.09 \pm$ 4.10, $36.5 \pm 3.49$, respectively, and $P$-value $<0.05$ for both). However, gravid and parity did not differ significantly between groups ( $p$-value> 0.05 for both). The maternal characteristics of the participants are compared in Table. 1.

Preeclampsia, diabetes mellitus, maternal hemoglobin, maternal hematocrit, and $\mathrm{C} / \mathrm{S}$ were higher in the chorangiosis group $\mathrm{OR}=1.6,95 \% \mathrm{C}$. I $(1.03-2.49)$, $\mathrm{OR}=3.98$, $95 \%$ C. I $(2.13-4.45), \mathrm{OR}=1.68,95 \%$ C. I $(1.44-1.96)$, $\mathrm{OR}=1.92,95 \%$ C. I $(1.13-1.26)$, and $\mathrm{OR}=2.1,95 \%$ C.I(1.33-3.33), respectively. However, hypertension, and other maternal diseases were not significantly different between the two groups ( $p$-value $>0.05$ for all).

The fetal features of 308 cases from the chorangiosis group and those of the control group are provided in Table. 2.

The amniotic fluid index was lower in the chorangiosis group $\mathrm{OR}=0.31,95 \%$ C. I (0.18-0.54), and oligohydramnios cases were higher in the chorangiosis group $\mathrm{OR}=$ 4.47, 95\% C. I (2.29-8.72). Moreover, polyhydramnios cases did not differ between the groups ( $p$-value>0.05). Fetal anomaly cases were higher in the chorangiosis group $\mathrm{OR}=4.22$, 95\% C. I (1.49-11.94). However, abnormal karyotype in fetuses did not differ between the groups ( $p$-value>0.05).

The perinatal and neonatal features of 308 babies from the chorangiosis group and those of the babies from the control group are provided in Table. 3 .

Gender did not differ between the groups. However, dead neonates and NICU admissions were higher in the chorangiosis group $\mathrm{OR}=2.9,95 \%$ C. I $(1.58-5.28)$, and $\mathrm{OR}=2.46,95 \%$ C. I (1.62-3.72), respectively. Moreover, birth weight, $\mathrm{PH}$ amount, 1st Apgar score, and 5th Apgar score were lower in the chorangiosis group, $\mathrm{OR}=$ $1,95 \%$ C. I $(0.999-1)$, OR $=0.097$, 95\% C. I $(0.011-0.86)$, $\mathrm{OR}=0.83,95 \%$ C.I $(0.76-0.90)$, and $\mathrm{OR}=0.85,955$ C.I(0.78-0.94), respectively.

Placenta, decidua, membrane, and cord histopathological examination of 308 chorangiosis cases and the control group are presented in Table. 4.

In total, a relationship was seen between the placenta location and chorangiosis status $(p$-value $<0.001)$. The fundal placenta was higher in the chorangiosis group $\mathrm{OR}=2.1,95 \%$ CI (1.15-3.88). Posterior placenta, however, was lower OR $=0.51,95 \%$ C.I $(0.37-0.71)$, and anterior placenta, lateral placenta, placenta previa did not differ significantly $(p$-value $>0.05$, for all). Perinatal outcomes are compared between the chorangiosis and control groups by placental site in Table 5 .

In posterior and anterior placenta cases, IUGR, C/S, neonatal death, and NICU were higher in the chorangiosis group compared to the control group $\mathrm{OR}=3.1,95 \%$ C.I $(1.67-5.75)$ and $\mathrm{OR}=4.28,95 \%$ C.I $(1.87-9.81)$; OR = 
Table 1 Maternal features of 308 chorangiosis cases and the control group from April 2014 to March 2018

\begin{tabular}{|c|c|c|c|}
\hline Baseline characteristics & Chorangiosis group $(n=308)$ & Control group $(n=308)$ & $P$-value \\
\hline Maternal age (Mean \pm SD) & $29.4 \pm 5.82$ & $30.3 \pm 5.48$ & 0.04 \\
\hline Gestational age (Mean \pm SD) & $35.09 \pm 4.10$ & $36.5 \pm 3.49$ & $<0.001$ \\
\hline Gravidity (Mean \pm SD) & $2.26 \pm 1.33$ & $2.32 \pm 1.29$ & 0.58 \\
\hline Parity (Mean \pm SD) & $1.42 \pm 0.82$ & $1.43 \pm 0.73$ & 0.89 \\
\hline \multicolumn{4}{|l|}{ Maternal diseases (n, \%) } \\
\hline Hypertension & $44(14.3 \%)$ & $32(10.4 \%)$ & 0.14 \\
\hline Preeclampsia & $58(18.8 \%)$ & $39(12.7 \%)$ & 0.03 \\
\hline Diabetes mellitus & $50(16.2 \%)$ & $15(4.9 \%)$ & $<0.001$ \\
\hline \multicolumn{4}{|l|}{ Other diseases ( $n, \%)$} \\
\hline Depression & $3(7.1 \%)$ & $2(10 \%)$ & 0.92 \\
\hline Hypothyroid & $24(57.1 \%)$ & $13(65 \%)$ & \\
\hline Minor thalassemia & $6(14.3 \%)$ & $2(10 \%)$ & \\
\hline Multiple sclerosis & $1(2.4 \%)$ & 0 & \\
\hline Asthma & $1(2.4 \%)$ & 0 & \\
\hline Lexi Fredrich syndrome & $1(2.4 \%)$ & 0 & \\
\hline Epilepsy & $1(2.4 \%)$ & 0 & \\
\hline Systemic Lupus Erythematosus & $5(11.9 \%)$ & $3(15 \%)$ & \\
\hline \multicolumn{4}{|l|}{ Hematological indices (Mean \pm SD. } \\
\hline Hemoglobin & $12.33 \pm 1.36$ & $11.71 \pm 0.88$ & $<0.001$ \\
\hline Hematocrit & $36.58 \pm 3.58$ & $35.04 \pm 2.47$ & $<0.001$ \\
\hline \multicolumn{4}{|l|}{ Rout of delivery (n, \%) } \\
\hline $\mathrm{C} / \mathrm{S}$ & 274 (89.0\%) & 239(77.6\%) & $<0.001$ \\
\hline
\end{tabular}

2.16, 95\% C.I $(1.23-3.87)$ and $\mathrm{OR}=2.14$, 95\% C.I $(1.42-$ 14.99), $\mathrm{OR}=2.04,95 \% \mathrm{C} . \mathrm{I}(1.2-4.23)$ and $\mathrm{OR}=5.81,95 \%$ C.I(1.66-20.39), OR $=2.7,95 \%$ C. I (1.69-4.13) and OR = 4.13, 95\% C.I(2.2-7.73), respectively. However, they did not differ in fundal placenta, lateral placenta, and placental perivia groups( $p$-value $>0.05$ for all). In posterior, anterior, and fundal placenta cases, the first minute Apgar score was lower in the chorangiosis group in comparison to that of the control group OR $=0.87,95 \%$ C.i(0.78-0.97), OR =0.66, 95\% C.I(0.54-0.8), and OR = 0.38 , 95\% C.I(0.15-0.95). Moreover, just in anterior and fundal placenta cases, the fifth minute Apgar score was lower in the chorangiosis group $\mathrm{OR}=0.65,95 \%$
C.i $(0.52-0.82)$, and $\mathrm{OR}=0.41,95 \%$ C. I (0.16-0.98) respectively.

Retro placental hemorrhage, perivillous fibrin deposition, and calcification were higher in the chorangiosis group OR $=11.8,95 \% \mathrm{CI}(6.5-21.45), \mathrm{OR}=19.96,955 \mathrm{CI}$ (7.12-55.98), and $\mathrm{OR}=4.05,95 \%$ CI (1.82-9.01), whereas accessory placenta and large placenta did not differ between the groups. Acute chorioamnionitis was higher in the chorangiosis group $\mathrm{OR}=6.38,95 \% \mathrm{C}$. I (2.62-15.49). However, meconium staining did not differ significantly. No significant difference was seen in terms of decidua and cord pathology between the groups. Kappa statistics for chorangiosis reporting by two

Table 2 Fetal features of 308 chorangiosis cases and the control group from April 2014 to March 2018

\begin{tabular}{|c|c|c|c|}
\hline Fetal feature & Chorangiosis group $(n=308)$ & Control group $(n=308)$ & $P$-value \\
\hline IUGR $(n, \%)^{a}$ & $79(25.6 \%)$ & $27(8.8 \%)$ & $<0.001$ \\
\hline \multicolumn{4}{|l|}{ Amniotic fluid index (n, \%) } \\
\hline Normal amniotic fluid index & 246(79.9\%) & 288(93.5\%) & $<0.001$ \\
\hline Oligohydramnios & $54(17.5 \%)$ & $12(13.9 \%)$ & \\
\hline Polyhydramnios & $8(2.6 \%)$ & $8(2.6 \%)$ & \\
\hline Fetal anomaly (n, \%) & $29(9.4 \%)$ & $7(2.3 \%)$ & $<0.001$ \\
\hline Abnormal karyotype in fetus ( $n, \%)$ & $2(0.6 \%)$ & 0 & 0.09 \\
\hline
\end{tabular}

${ }^{a}$ Intra Uterine Growth Restriction (IUGR) is an estimated fetal weight below the 10th percentile for gestational age, based on sonography 
Table 3 Perinatal and neonatal features of 308 babies from the chorangiosis and control groups from April 2014 to March 2018

\begin{tabular}{|c|c|c|c|}
\hline Perinatal \& neonatal feature & Chorangiosis group $(n=308)$ & Control group $(n=308)$ & $P$-value \\
\hline \multicolumn{4}{|l|}{ Gender (n, \%) } \\
\hline Male & 179(58.1\%) & $177(57.5 \%)$ & 0.87 \\
\hline \multicolumn{4}{|l|}{ Survival (n, \%) } \\
\hline Dead & $42(13.6 \%)$ & $17(5.5 \%)$ & $<0.001$ \\
\hline Birth weight $(\mathrm{Kg})($ mean \pm s.d) & $2372.06 \pm 911.4$ & $2780.34 \pm 867.365$ & $<0.001$ \\
\hline Actual birth weight $<10$ th percentile(n, \%) & $38(12.3 \%)$ & $21(6.8 \%)$ & 0.02 \\
\hline PH amount (mean \pm s.d) & $7.24 \pm .09$ & $7.25 \pm 0.06$ & 0.04 \\
\hline Apgar score at 1 min (mean \pm s.d) & $7.09 \pm 2.56$ & $8.17 \pm 1.84$ & $<0.001$ \\
\hline Apgar score at 5 min (mean \pm s.d) & $8.42 \pm 2.55$ & $9.30 \pm 1.74$ & $<0.001$ \\
\hline NICU Admission (n, \%) & $140(45.5 \%)$ & $68(22.1 \%)$ & $<0.001$ \\
\hline
\end{tabular}

pathologists was estimated, showing a high agreement of 95\%. Moreover, the power of the study was estimated at almost 99\%.

\section{Discussion}

The etiology of chorangiosis is not well-known, but it is hypothesized that chronic hypoperfusion or tissue hypoxemia is the leading cause of placenta chorangiosis. According to this theory, hypoxia induces vascular growth factors expression, probably leading to an excessive villous capillary growth and a high proliferative activity of connective tissue $[4,19]$. In this study, we reviewed the pathology results of all placentas sent to the Pathology Department over a fouryear period to look for evidence of chorangiosis.

Table 4 Placenta, decidua, membrane, and cord histopathological examination and placental site of 308 chorangiosis cases and the control group from April 2014 to March 2018

\begin{tabular}{|c|c|c|c|}
\hline Placental Features & Chorangiosis group $(n=308)$ & Control group $(n=308)$ & $P$-value \\
\hline \multicolumn{4}{|l|}{ Placenta site $^{a}$} \\
\hline Posterior placenta & $141(45.8 \%)$ & 187(60.7\%) & \multirow[t]{5}{*}{0.001} \\
\hline Anterior placenta & $117(38.0 \%)$ & $99(32.1 \%)$ & \\
\hline Lateral placenta & $11(3.6 \%)$ & $0(0 \%)$ & \\
\hline Fundal placenta & $34(11.0 \%)$ & $18(5.8 \%)$ & \\
\hline Placenta previa & $5(1.6 \%)$ & $4(1.3 \%)$ & \\
\hline \multicolumn{4}{|l|}{ Placenta Pathology } \\
\hline Retro Placental Hemorrhage & 107(34.7\%) & $14(4.5 \%)$ & \multirow[t]{5}{*}{$<0.001$} \\
\hline Perivillous Fibrin deposition & $66(21.4 \%)$ & $4(1.3 \%)$ & \\
\hline Calcification & $32(10.4 \%)$ & $8(2.6 \%)$ & \\
\hline Accessory placenta & $1(0.3 \%)$ & 0 & \\
\hline Large placenta & $9(2.9 \%)$ & $3(1 \%)$ & \\
\hline \multicolumn{4}{|l|}{ Decidua Pathology } \\
\hline Inflamed & $4(13.3 \%)$ & $1(20 \%)$ & \multirow[t]{3}{*}{0.85} \\
\hline Thick wall blood vessel & $1(3.3 \%)$ & 0 & \\
\hline Calcification & 25(83.3\%) & $4(80 \%)$ & \\
\hline \multicolumn{4}{|l|}{ Membrane Pathology } \\
\hline Meconium staining & $8(17.8 \%)$ & $7(53.8 \%)$ & \multirow[t]{2}{*}{0.009} \\
\hline Acute chorioamnionitis & $37(92.2 \%)$ & $6(46.2 \%)$ & \\
\hline \multicolumn{4}{|l|}{ Cord Pathology } \\
\hline Single umbilical artery & $5(38.5 \%)$ & $2(66.7 \%)$ & \multirow[t]{3}{*}{0.56} \\
\hline Abnormal cord insertion & $5(38.5 \%)$ & $1(33.3 \%)$ & \\
\hline Acute funisitis & $3(23.1 \%)$ & 0 & \\
\hline
\end{tabular}

${ }^{\mathrm{a}}$ According to ultrasonography report 
Table 5 The comparison of perinatal outcomes of chorangiosis and control groups by placenta site

\begin{tabular}{|c|c|c|c|c|}
\hline Placental site & outcome & Chorangiosis group(n, \%) & Control group(n,\%) & $p$-value \\
\hline Posterior placenta & $\operatorname{IUGR}(N=187)$ & 169(90.4\%) & 18(9.6\%) & $<0.001$ \\
\hline \multirow[t]{5}{*}{ Posterior placenta Anterior placenta } & $C / S(N=141)$ & $122(86.5 \%)$ & $19(13.5 \%)$ & 0.009 \\
\hline & Neonatal death $(N=187)$ & $173(92.5 \%)$ & $14(7.5 \%)$ & 0.04 \\
\hline & $\operatorname{NICU}(N=141)$ & $75(53.2 \%)$ & $66(46.8 \%)$ & $<0.001$ \\
\hline & 1stminute Apgar score $($ mean \pm s.d) & $7.37 \pm 2.18$ & $7.97 \pm 2.05$ & 0.012 \\
\hline & $\operatorname{IUGR}(N=117)$ & $85(72.6 \%)$ & $32(27.4 \%)$ & $<0.001$ \\
\hline \multirow[t]{6}{*}{ Anterior placenta Fundal placenta } & $C / S(N=117)$ & $106(90.6 \%)$ & $11(9.4 \%)$ & 0.05 \\
\hline & Neonatal death $(N=117)$ & $99(84.6 \%)$ & 18(15.4\%) & 0.002 \\
\hline & $\operatorname{NICU}(N=117)$ & $61(52.1 \%)$ & $56(47.9 \%)$ & $<0.001$ \\
\hline & $1^{\text {st }}$ minute Apgar score $($ mean \pm s.d) & $6.77 \pm 2.84$ & $8.44 \pm 1.55$ & $<0.001$ \\
\hline & 5th minute Apgar score (mean \pm s.d) & $8.07 \pm 2.77$ & $9.50 \pm 1.55$ & $<0.001$ \\
\hline & 1st minute Apgar score $($ mean \pm s.d) & $6.71 \pm 3.14$ & $8.71 \pm 0.46$ & 0.01 \\
\hline Fundal placenta & 5th minute Apgar score (mean $\pm s . d)$ & $7.9 \pm \pm 3.3$ & $9.77 \pm 0.42$ & 0.001 \\
\hline
\end{tabular}

In our study, the gestational age was significantly lower in the chorangiosis group. Caldarella et al. [20] showed that chorangiosis generally occurs at term, but no increases have been reported to occur at any gestational age. Nevertheless, some authors have reported chorangiosis is more common after 37 weeks of pregnancy [21]. Another study by GÜN et al. [22] found that chorangiosis had been correlated with increased preterm delivery.

Regarding hematological parameters, our study has also identified that the mean of hemoglobin and hematocrit in women with chorangiosis were significantly more than that of the control group. Although anemia can cause chorangiosis $[5,23]$, several other conditions contributing to chronic long-standing placental hypoperfusion or low-grade tissue hypoxemia may also lead to chorangiosis. Suzuki et al. [24] revealed that low efficiency of oxygen transfer from maternal to fetal circulation, irrespective of maternal condition, results in chorangiosis as it tends to facilitate vascular remodeling to make up for low oxygen supply. They described a case of diamniotic dichorionic twins, only one of whom manifested growth restriction with placental chorangiosis.

The association of chorangiosis with maternal and placental pathological conditions such as preeclampsia, diabetes mellitus, drug ingestion, urinary tract infections, placental abruption, villitis, and umbilical cord anomalies were also previously noted [20, 22, 25].

In agreement with the previous reports, our results demonstrated that some maternal diseases such as preeclampsia and diabetes mellitus were strongly associated with chorangiosis, while other diseases such as depression, multiple sclerosis, hypothyroidism, minor thalassemia, asthma, epilepsy, and systemic lupus erythematous were not. In this regard, Gun and colleagues reported that out of the ten cases with chorangiosis, two patients had preeclampsia, two had thyroid dysfunction, and one patient had multiple sclerosis [22].

It should be noted that diabetes mellitus is a type of chronic oxidative stress (preuterine pattern). Glycemia affects the capillary of chorionic villi by insulin ephrinB2 expression, a signaling molecule implicated in sprouting. Preeclampsia also is a state of placental chronic hypoxia with a uterine pattern with an early onset. Serum biomarkers of preeclampsia have been detected as early as 7 weeks of gestational age. Maldevelopment of spiral arteries in the first trimester results in placental malfunction and hence poor pregnancy outcomes [5].

In the present study, the fundal placenta appeared to be higher in the chorangiosis group, and the posterior placenta was found to be lower, while the anterior placenta, lateral placenta, and placenta previa did not vary significantly. In posterior and anterior placenta cases, IUGR, C/S, neonatal death, and NICU were higher in the chorangiosis group, but not different in the fundal placenta, lateral placenta, and placental previa groups. In posterior, anterior, and fundal placenta cases, the first minute Apgar score was lower in the chorangiosis group. No specific study was found to report on the association between chrongeiosis and the placental location. However, some studies were done on the association between placental location and pregnancy outcome.

Placenta location is strongly associated with preeclampsia, causing fetal distress during labor, IUGR, and unfavorable pregnancy outcomes [26, 27]. Zia S. found that anterior placental implantation increases the risk of hypertension, gestational diabetes mellitus, placental abruption, intrauterine growth retardation, and intrauterine fetal death induced by pregnancy. Posterior placenta has a significant association with preterm labor 
[28]. However, Granfors M. et al. reported that fundal and lateral placental locations, as compared with posterior placental location, are linked to preterm birth, smallfor-gestational-age birth, as well as increased manual removal of the placenta in nulliparous women who had vaginal deliveries [29].

Additionally, the rate of cesarean sections in cases with chorangiosis was significantly more than that in the control group. This finding was along with the results obtained from previous studies [25, 30]. It is worth mentioning, however, that the present study was done in a tertiary referral center, and that is why the cesarean sections rate is relatively high in both groups.

Placental lesions are considered as one of the main contributors to neonatal mortality and morbidity. Among the placental lesions, those consistent with maternal vascular malperfusion, such as chorangiosis, have the most significant role to play in adverse fetal outcomes [31]. According to our results, neonatal morbidity and mortality in the chorangiosis population such as low Apgar (min 1 and 5), low birth weight, low PH in arterial blood gas, fetal anomaly, death after birth, and NICU care were higher than those in mothers without chorangiosis. Another important aspect of our findings has to do with the association of chorangiosis with IUGR, oligohydramnios, and anterior placenta. Therefore, chorangiosis is associated with high neonatal morbidity and mortality. This is concordant with the results of all studies on chorangiosis, which reported a higher incidence of neonatal morbidity and mortality in chorangiosis population. Gupta and colleagues showed that neonatal morbidity (IUGR, low birth weight, prematurity, and NICU admissions) were high in 15 cases diagnosed with chorangiosis, and there was a strong positive association of chorangiomatous lesions with pregnancy-induced hypertension/preeclampsia [25]. Moreover, another study performed by Gun and colleagues revealed that preterm birth, low Apgar, and NICU admission were most common in mothers with chorangiosis [22].

Our study has also identified that the incidence of other placental abnormalities (such as fibrin deposition, calcification, and retro placenta hemorrhage), decidua, membrane, and umbilical cord abnormalities in the chorangiosis group was significantly more than that in women without chorangiosis. This is similar to what was reported by Gun et al. [22], who showed that placental calcification, perivillous fibrin, chorioamnionitis, umbilical cord knots, intervillous hemorrhage, and umbilical vein dilatation were commonly observed placental findings in chorangiosis cases. This might be due to the low efficiency of oxygen transfer and maternal vascular malperfusion.

A large sample size study investigating various outcomes and pregnancy characteristics has strengthened the current research, thus providing more accurate statistics, identifying outliers that could skew the data, and lowering the margin of error. It helped control on exogenous variables and latent confounders, thereby enhancing the power of the study. Moreover, a broad clinical and histopathological association with chorangiosis was investigated in the present study. However, a retrospective design left us with no choice but investigate only cases with pregnancy complications without including normal pregnancies, which is a limitation in the study. Choosing a control group with other placental pathological diagnoses was because, in line with the guidelines, only placentas with certain indications are routinely referred to the histopathology lab, and we had to select from other diagnostic pathology complications to examine chorangiosis.

\section{Conclusion}

The findings of our study suggest that chorangiosis should be considered as a hypoxia-associated placental lesion that may occur in association with several maternal diseases such as preeclampsia and diabetes mellitus. In conclusion, although chorangiosis is a rare condition, it is associated with higher incidences of perinatal and neonatal morbidity and mortality. Therefore, it should be considered as a meaningful clinical sign of adverse pregnancy outcomes, and should be mentioned in the pathology evaluation.

\section{Abbreviations}

GA: Gestational Age; NICU: Neonatal Intensive Care Unit; AFI: Amniotic Fluid Index; IUGR: Intra-Uterine Growth Retardation; SD: Standard Deviation

\section{Acknowledgments}

This article was extracted from a thesis written by Zinat Karimi for the degree of Obstetrics and Gynecology Specialty. The authors would like to thank Shiraz University of Medical Sciences for their financial support of the study.

\section{Authors' contributions}

HV and MAJ contributed to study design, data interpretation, and critical revision of the manuscript. FA contributed to data collection, data interpretation, and revision of the manuscript. ZK contributed to data collection, data interpretation, drafting of the manuscript. All authors have read and approved the final manuscript.

\section{Funding}

The study was financed and supported by the Research Vice-chancellor of Shiraz University of Medical Sciences (grant No. 12737).

\section{Availability of data and materials}

The data of this study are accessible by directly contacting the corresponding author at mojganakbarzadeh@yahoo.com

\section{Ethics approval and consent to participate}

The study protocol was approved by the institutional review board (IRB) of Shiraz University of Medical Sciences, and the approval of the Ethics Committee (IR.SUMS.MED.REC.1396.s294) was achieved before the study began.

Consent for publication

Not Applicable. 


\section{Competing interests}

The authors declare no competing interests.

\section{Author details}

'Maternal-Fetal Medicine Research Center, Shiraz University of Medical Sciences, Shiraz, Iran. ${ }^{2}$ Obstetrics \& Gynecology department, School of Medicine, Shiraz University of Medical Sciences, Shiraz, Iran. ${ }^{3}$ Pathology Department, School of Medicine, Shiraz University of Medical Sciences, Postal code/ P.O. Box: 34786-71946, Shiraz, Iran. ${ }^{4}$ Department of Medical Laboratory Sciences, School of Paramedical Sciences, Shiraz University of Medical Sciences, Shiraz, Iran

Received: 29 April 2020 Accepted: 20 January 2021

Published online: 30 January 2021

\section{References}

1. Altshuler G. A conceptual approach to placental pathology and pregnancy outcome. Semin Diagn Pathol. 1993;10(3):204-21.

2. Dureau ZJ, Rogers BB. Placental pathology. Diagn Histopathol. 2019;25(9): 341-9.

3. Spencer MK, Khong TY. Conformity to guidelines for pathologic examination of the placenta: rates of submission and listing of clinical indications. Arch Pathol Lab Med. 2003;127(2):205-7.

4. De La Ossa MM, Cabello-Inchausti B, Robinson MJ. Placental chorangiosis. Arch Pathol Lab Med. 2001;125(9):1258.

5. Stanek J. Hypoxic patterns of placental injury: a review. Arch Pathol Lab Med. 2013;137(5):706-20.

6. Schwartz DA. Chorangiosis and its precursors: underdiagnosed placental indicators of chronic fetal hypoxia. Obstet Gynecol Surv. 2001;56(9):523-5.

7. Soma H, Hata T, Oguro T, Fujita K, Kudo M, Vaidya U. Characteristics of histopathological and ultrastructural features of placental villi in pregnant Nepalese women. Med Mol Morphol. 2005;38(2):92-103.

8. Altshuler G. Chorangiosis. An important placental sign of neonatal morbidity and mortality. Arch Pathol Lab Med. 1984;108(1):71-4

9. Ogino S, Redline RW. Villous capillary lesions of the placenta: distinctions between chorangioma, chorangiomatosis, and chorangiosis. Hum Pathol. 2000;31(8):945-54.

10. Stanek J. Diagnosing placental membrane hypoxic lesions increases the sensitivity of placental examination. Arch Pathol Lab Med. 2010;134(7):989-95.

11. Stanek J. Placental membrane and placental disc microscopic chorionic cysts share similar clinicopathologic associations. Pediatr Dev Pathol. 2011; 14(1):1-9.

12. Stanek J. Placental hypoxic overlap lesions: a clinicoplacental correlation. J Obstet Gynaecol Res. 2015:41(3):358-69.

13. Stanek J, Biesiada J. Sensitivity and specificity of finding of multinucleate trophoblastic giant cells in decidua in placentas from high-risk pregnancies. Hum Pathol. 2012;43(2):261-8.

14. Stanek J, Weng E. Microscopic chorionic pseudocysts in placental membranes: a histologic lesion of in utero hypoxia. Pediatr Dev Pathol. 2007:10(3):192-8.

15. Altshuler $\mathrm{G}$. Role of the placenta in perinatal pathology (revisited). Pediatr Pathol Lab Med. 1996;16(2):207-34.

16. Stanek J. Chorangiosis of chorionic villi: what does it really mean? Arch Pathol Lab Med. 2016;140(6):588-93.

17. Khong TY, Mooney EE, Ariel I, Balmus NC, Boyd TK, Brundler MA, Derricott H, Evans MJ, Faye-Petersen OM, Gillan JE, et al. Sampling and definitions of placental lesions: Amsterdam placental workshop group consensus statement. Arch Pathol Lab Med. 2016;140(7):698-713.

18. Battaglia FC, Lubchenco LO. A practical classification of newborn infants by weight and gestational age. J Pediatr. 1967;71(2):159-63.

19. Gupta R, Nigam S, Arora P, Khurana N, Batra S, Mandal AK. Clinicopathological profile of 12 cases of chorangiosis. Arch Gynecol Obstet. 2006; 274(1):50-3.

20. Caldarella A, Buccoliero AM, Taddei GL. Chorangiosis: report of three cases and review of the literature. Pathol Res Pract. 2003;199(12):847-50.

21. Lisboa Bittencourt A, Chagas K, Teixeira Calabrick VA. Large placental hemangioma diagnosed by ultrasonography--a case report. Sao Paulo Med J. 1995;113(6):1033-5.

22. Gün BD, Barut F, Tanrıverdi A, Özdamar ŞO, Barut A. Placental Chorangiosis: an important pattern of placental injury. Gynecol Obstet Reprod Med. 2006; 12(3):176-9.
23. Vafaei $H$, Karimi S, Akbarzadeh Jahromi M. The effect of mother's $\beta$ thalassemia minor on placental histology and neonatal outcomes; 2020. p. $1-8$.

24. Suzuki K, Itoh H, Kimura S, Sugihara K, Yaguchi C, Kobayashi Y, Hirai K, Takeuchi K, Sugimura M, Kanayama N. Chorangiosis and placental oxygenation. Congenit Anom. 2009:49(2):71-6.

25. Gupta D, Singh P. Chorangiosis of the placenta: association with maternal profile and neonatal outcome, experience at our centre. J Med Sci Clin Res JMSCR. 2018;06(2):1184-7. https://doi.org/10.1186/s12884-021-03576-0/.

26. Kakkar T, Singh V, Razdan R, Digra SK, Gupta A, Kakkar M. Placental laterality as a predictor for development of preeclampsia. J Obstet Gynaecol India. 2013;63(1):22-5

27. Kofinas AD, Penry M, Swain M, Hatjis CG. Effect of placental laterality on uterine artery resistance and development of preeclampsia and intrauterine growth retardation. Am J Obstet Gynecol. 1989;161(6 Pt 1):1536-9.

28. Zia S. Placental location and pregnancy outcome. J Turk Ger Gynecol Assoc. 2013;14(4):190-3.

29. Granfors M, Stephansson O: Placental location and pregnancy outcomes in nulliparous women: A population-based cohort study. 2019, 98(8):988-996.

30. Petersen SS, Khangura R, Davydov D, Zhang Z, Sangha R. Placental Chorangiosis: increased risk for cesarean section. Case Rep Obstet Gynecol. 2017:2017:5610945.

31. Kidron D, Bernheim J, Aviram R. Placental findings contributing to fetal death, a study of 120 stillbirths between 23 and 40 weeks gestation. Placenta. 2009;30(8):700-4

\section{Publisher's Note}

Springer Nature remains neutral with regard to jurisdictional claims in published maps and institutional affiliations.
Ready to submit your research? Choose BMC and benefit from:

- fast, convenient online submission

- thorough peer review by experienced researchers in your field

- rapid publication on acceptance

- support for research data, including large and complex data types

- gold Open Access which fosters wider collaboration and increased citations

- maximum visibility for your research: over $100 \mathrm{M}$ website views per year

At BMC, research is always in progress.

Learn more biomedcentral.com/submissions 